\title{
A simple model for cloud radiative forcing
}

\author{
T. Corti and T. Peter \\ Institute for Atmospheric and Climate Science, Swiss Federal Institute of Technology (ETH), Zurich, Switzerland
}

Received: 23 February 2009 - Published in Atmos. Chem. Phys. Discuss.: 31 March 2009

Revised: 16 July 2009 - Accepted: 20 July 2009 - Published: 12 August 2009

\begin{abstract}
We present a simple model for the longwave and shortwave cloud radiative forcing based on the evaluation of extensive radiative transfer calculations, covering a global range of conditions. The simplicity of the model equations fosters the understanding on how clouds affect the Earth's energy balance. In comparison with results from a comprehensive radiative transfer model, the accuracy of our parameterization is typically better than $20 \%$. We demonstrate the usefulness of our model using the example of tropical cirrus clouds. We conclude that possible applications for the model include the convenient estimate of cloud radiative forcing for a wide range of conditions, the evaluation of the sensitivity to changes in environmental conditions, and as a tool in education. An online version of the model is available at http://www.iac.ethz.ch/url/crf.
\end{abstract}

\section{Introduction}

Clouds are an important element of the climate system as they have a big impact on the Earth's energy balance. The role of clouds for the climate system can be described using the concept of radiative forcing. Here, we define cloud radiative forcing (CRF) as change in radiative fluxes at the tropopause in the presence of a cloud relative to the clear sky situation, in accordance with Chylek and Wong (1998) and Chen et al. (2000).

Several studies have quantified the CRF (e.g., Barkstrom, 1984; Rossow and Lacis, 1990; Hartmann et al., 1992). Chen et al. (2000) have estimated shortwave and longwave CRF to amount to approximately -50 and $+20 \mathrm{Wm}^{-2}$, respectively, on the global and annual mean. This results in a negative net CRF of $-30 \mathrm{Wm}^{-2}$, which means that clouds have an over-

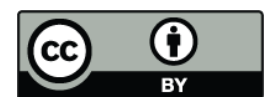

Correspondence to: T. Corti (thierry.corti@env.ethz.ch) all cooling effect for the Earth-atmosphere system. This is however not true for all individual cloud types. Most importantly, cirrus clouds have an overall positive radiative impact (Chen et al., 2000).

The importance of the CRF is contrasted by a general lack of simplified concepts explaining what determines the sign and magnitude of CRF for different types of clouds and environmental conditions. Previous treatments of CRF have been either relatively complex or of specialized applicability (e.g., Baker, 1997; Chylek and Wong, 1998; Hartmann et al., 2001).

Here, we present a simple but nevertheless quantitative model for CRF. We discuss the longwave and shortwave CRF, derive simple approximations and use results from radiative transfer calculations to determine the free parameters of our parameterizations. Even though the resulting equations are simple, they provide useful insight into the way clouds influence the Earth's radiative balance. In order to demonstrate the capabilities of our parameterizations, we apply them to the case of tropical cirrus clouds.

\section{Method}

We derive a simple parametrization for the longwave and shortwave CRF with a few free parameters, which we estimate from calculations with the Fu-Liou radiative transfer model (Fu and Liou, 1992, 1993). These calculations cover a global range of realistic atmospheric conditions and cloud properties, in order to obtain a parameterization with broad applicability.

Two years (2000 and 2001) of monthly and longitudinal mean atmospheric profiles of temperature, ozone and water vapor data with $2.5^{\circ}$ latitudinal resolution from the ECMWF ERA 40 reanalysis project are used to describe the variability in atmospheric conditions. All profiles are interpolated to a vertical resolution of $250 \mathrm{~m}$. The surface properties are

Published by Copernicus Publications on behalf of the European Geosciences Union. 
characterized by corresponding ERA 40 monthly and longitudinal mean surface temperature and albedo.

Radiative transfer calculations are performed for each atmospheric condition in combination with a set of sun elevations corresponding to a time step of three hours during daytime. First, a clear sky calculation is accomplished to define the reference condition for the calculation of CRFs. Then, a set of calculations is performed, inserting a cloud layer with $1 \mathrm{~km}$ vertical extent and an optical depth between 0.01 and 100 at a height ranging from the boundary layer to the tropopause. Ice clouds are assumed at temperatures below $-10^{\circ} \mathrm{C}$ and water clouds above. Ice particle sizes are chosen based on Heymsfield and Platt (1984), depending on the cloud top temperature, assuming that all ice crystals are hexagonal columns (Fu, 1996; Fu et al., 1998). For water clouds, the effective particle radius is set to $10 \mu \mathrm{m}$. CRF is then calculated from changes in longwave and shortwave radiative fluxes at the troposphere. Altogether, several hundred thousand radiative calculations are performed in this manner.

It is important to note that we define CRF relating to the difference between two local atmospheric conditions. This definition is consistent with Chylek and Wong (1998) and Chen et al. (2000). It differs however from the definition in IPCC (2007), where radiative forcing is defined on a global level and relative to preindustrial conditions. Moreover, we define radiative forcing as the change in net irradiance at the tropopause without allowing for stratospheric temperatures to readjust to radiative equilibrium. We thus calculate CRF as an "instantaneous radiative forcing", whereas other definitions include the adjustment of stratospheric temperatures or further elements of the climate system (IPCC, 2007).

We will apply our simple model for CRF to the case of tropical cirrus clouds (Sect. 4). To do so, we use a mean temperature, water vapor and ozone profile from tropical balloon sonde measurements (Corti et al., 2005) to describe the atmospheric properties and perform calculations as described above to evaluate the performance of our parameterization.

\section{Cloud radiative forcing}

We derive a simple model for CRF and use results from radiative transfer calculations to determine the few free parameters of our parameterizations. The main assumptions made in the derivation of the equations and required input parameters are summarized in Table 1, including the approximate range of applicability based on the range of parameters used in the radiative transfer calculations.

\subsection{Longwave radiative budget}

Longwave radiation is defined as radiation with wavelengths longer than $4 \mu \mathrm{m}$ and is essentially of terrestrial origin (Glickman, 2000). As a consequence, the longwave radiative budget at the top of the atmosphere (TOA) is composed

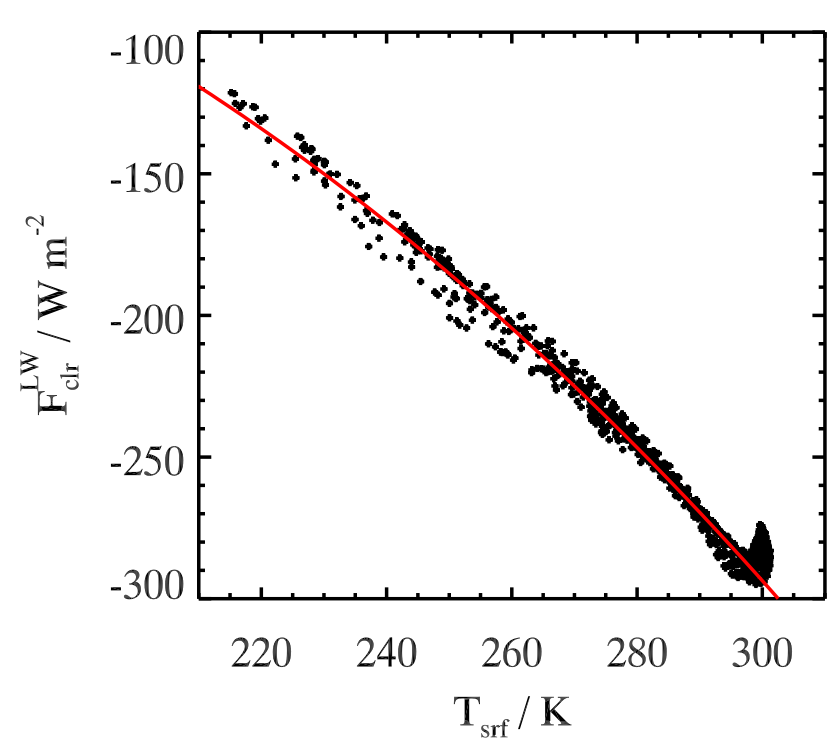

Fig. 1. Clear sky longwave radiative flux at TOA $\left(F_{\mathrm{clr}}^{L W}\right)$ as function of surface temperature $T_{\text {srf }}$ based on global radiative transfer calculations (symbols) and following the parametrization in Equation 2 (red curve).

of upwelling fluxes only, because there are no significant incoming longwave radiative fluxes originating from the sun. If we assumed the Earth to be a black body at a uniform temperature $T_{\text {srf }}$ without surrounding atmosphere, the net longwave radiative flux at TOA $\left(F^{L W}\right)$ would correspond to the outgoing longwave radiation (OLR) emitted by the Earth following the Stefan-Boltzmann law,

$F^{L W}=\sigma T_{\text {srf }}^{4}$,

where $\sigma$ is the Stefan-Boltzmann constant and $T_{\text {srf }}$ the Earth's surface temperature. In reality however, the Earth is not a black body and has an atmosphere that absorbs and emits longwave radiation. The absorption is highly wavelength dependent and determined by the atmospheric composition, most importantly by water vapor and carbon dioxide $\left(\mathrm{CO}_{2}\right)$ (Liou, 1992). Due to its long atmospheric lifetime, the $\mathrm{CO}_{2}$ concentration is roughly constant all over the globe. The water vapor concentrations in contrast vary considerably. The atmospheric water vapor path is closely linked to the temperature in the lower troposphere via the ClausiusClapeyron relation and thus correlated with the Earth's surface temperature.

The symbols in Figure 1 show calculated clear sky longwave radiative flux at TOA $F_{\text {clr }}^{L W}$, using the radiative transfer model in combination with ECMWF ERA-40 profiles. The longwave radiative fluxes are plotted as negative values to emphasize that it represents an energy loss for the Earthatmosphere system. 
Table 1. Main assumptions for the CRF model and necessary input parameters with approximate range of applicability.

\begin{tabular}{lll}
\hline & Longwave CRF & Shortwave CRF \\
\hline Assumptions & Plane parallel model & Plane parallel model \\
& Clear sky radiation depends on & Atmospheric absorption only above \\
& surface temperature only & Noud absorbing cloud \\
& Cloud is semi-transparent black body & Isotropic upward radiation \\
& Cloud emissivity depends on optical & Istepth $\tau(\leq 100)$ \\
depth only & Optical depth \\
Input parameters & Optical depth $\tau(\leq 100)$ & Surface albedo $\alpha(0.0-1.0)$ \\
& Cloud temperature $T_{\text {cld }}(180 \mathrm{~K}-305 \mathrm{~K})$ & Solar constant $S$ \\
& Surface temperature $T_{\text {srf }}(210 \mathrm{~K}-305 \mathrm{~K})$ & Zenith angle $Z\left(0^{\circ}-90^{\circ}\right)$ \\
& & Note: $I=S \cos (Z)$ \\
\hline
\end{tabular}

Assuming a plane parallel cloud free atmosphere, we can attempt to approximate $F_{\mathrm{clr}}^{L W}$ by

$F_{\mathrm{clr}}^{L W} \approx \sigma^{*} T_{\mathrm{srf}}^{k^{*}}$

where $\sigma^{*}$ and $k^{*}$ are two parameters which we can estimate from radiative calculations. We use the symbol * to highlight fitting coefficients of our approximations. A regression analysis applied to the computed fluxes leads to a best estimate for $\sigma^{*}=1.607 \times 10^{-4} \mathrm{~W} \mathrm{~m}^{-2} \mathrm{~K}^{-2.528}$ and $k^{*}=2.528$, resulting in the red curve in Fig. 1. The estimated values following this parametrization always remain within $10 \%$ from the values calculated using the radiative transfer model, with a mean error of $2.2 \%$ and a standard deviation of $5.6 \mathrm{Wm}^{-2}$. The most prominent deviations are found at the highest temperatures, where the variability of the atmospheric water vapor path dominates the variability in $F_{\mathrm{clr}}^{L W}$ (Raval et al., 1994). Therefore, the absolute value of $F_{\mathrm{clr}}^{L W}$ is lower in the moist inner tropics than in the remaining, drier tropics.

We could improve our parametrization by including a measure for the amount of absorption from water vapor, e.g., by adopting the parametrization by Allan et al. (1999), which takes the column averaged tropospheric relative humidity into account. But because we are more interested in cloud radiative effects than in a sophisticated parametrization of $F_{\mathrm{clr}}^{L W}$, we leave it at this very simple parametrization.

Next, we want to see how a cloud influences $F^{L W}$. We approximate a cloud by a semi-transparent black body. The cloud absorbs part of the upwelling longwave radiation originating from the Earth surface and transmits the remaining radiation. Part of the absorbed radiation is re-emitted by the cloud following the Stefan-Boltzmann law at the temperature of the cloud. Figure 2 illustrates the clear (left) and cloudy (right) sky situation. The transparency of the cloud can be described with the help of the emissivity $\epsilon$. The emissivity $\epsilon$ of a cloud is the ratio of the radiation emitted by the cloud to the radiation emitted by a black body at the same temperature. At the same time the infrared emissivity of a cloud

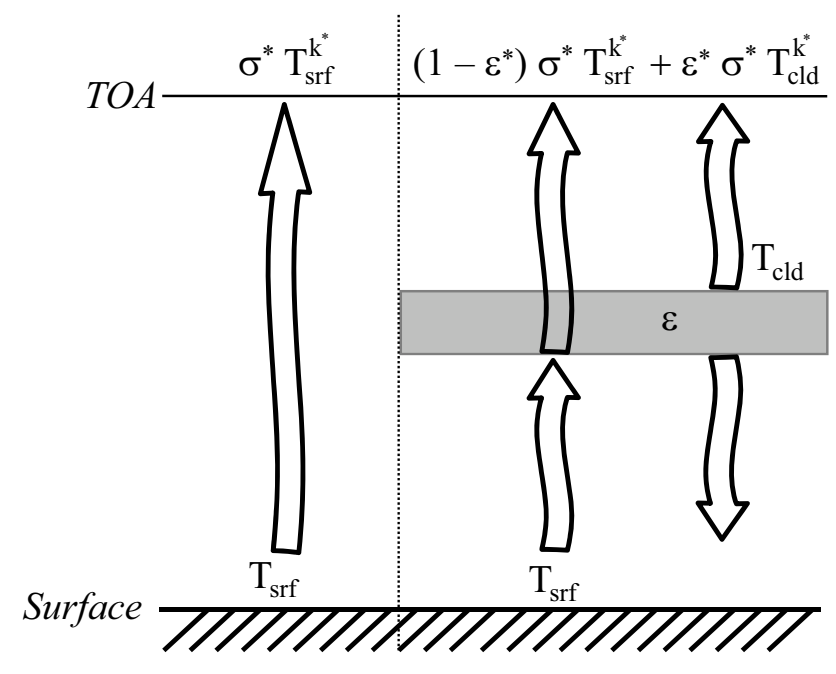

Fig. 2. Simplified plane parallel model to estimate the outgoing longwave radiation (ORL) in clear sky (left) and for a cloudy atmosphere (right). $\quad T_{\text {srf }}$ is the surface temperature, $T_{\text {cld }}$ the cloud top temperature, $\sigma^{*}$ the effective Stefan-Boltzmann constant in our parametrization and $\epsilon^{*}$ the effective cloud emissivity.

corresponds to its absorptivity based on Kirchhoff's law, i.e., the fraction of incident infrared radiation that is absorbed by the cloud.

We can thus express the cloudy sky $F^{L W}$ as

$F_{\mathrm{cld}}^{L W} \approx\left(1-\epsilon^{*}\right) \sigma^{*} T_{\mathrm{srf}}^{k^{*}}+\epsilon^{*} \sigma^{*} T_{\mathrm{cld}}^{k^{*}}$,

where $T_{c l d}$ is the cloud top temperature. The first term in Eq. (3) is the amount of $F_{\mathrm{clr}}^{L W}$ transmitted through the cloud, whereas the second term is the amount of longwave radiation emitted by the cloud. We have to notice that this parametrization is a crude approximation. Specifically, the parameters $\sigma^{*}$ and $k^{*}$ have been determined from clear sky calculations and do not necessarily apply to the radiation emitted by a 
cloud. We will take this into account in our calculation of the cloud emissivity, using an effective emissivity $\epsilon^{*}$. The advantage of Eq. (3) in this form is that it is very simple and leaves us only one parameter to adjust, which we can determine from radiative transfer calculations.

The cloud emissivity $\epsilon$ mainly depends on the cloud optical depth $\tau$, which is in turn a moderately wavelength dependent quantity. For the sake of simplicity, we will only use the shortwave optical depth in our parametrization. Following Stephens et al. (1990), the cloud emissivity $\epsilon$ is approximately

$\epsilon \approx 1-e^{-\delta \tau}$,

where $\delta=D\left(1-\tilde{\omega}_{o}\right)$ and $D(\approx 1.66)$ is the photon diffusivity and $\tilde{\omega}_{o}$ the single scattering albedo. Equation (4) is based on the assumption of zero scattering (Stephens et al., 1990).

With the help of our radiative calculations, we can now determine a typical value for $\delta$ to estimate the effective emissivity $\epsilon^{*}$ in Equation 3 as function of the shortwave cloud optical depth $\tau$. We find a typical value for $\delta, \delta^{*}=0.75$. We again use the symbol ${ }^{*}$ to emphasize that it is a parameter for our approximation. Our value for $\delta^{*}$ implies that $\tilde{\omega}_{o}=0.55$, which is realistic for longwave radiation (Stephens et al., 1990). The exact value of $\delta^{*}$ is not decisive for our parameterization, since variations of $10 \%$ in this parameter increases the mean error of $\mathrm{CRF}^{L W}$ (Eq. 5) in comparision with radiative transfer calculations only by about $1 \%$.

We can now calculate the longwave Cloud Radiative Forcing $\left(\mathrm{CRF}^{L W}\right)$ as the difference between $F_{\text {cld }}^{L W}$ and $F_{\mathrm{clr}}^{L W}$, this is, the difference in outgoing longwave radiation with the cloud present compared to the clear sky case:

$\mathrm{CRF}^{L W}=F_{\mathrm{cld}}^{L W}-F_{\mathrm{clr}}^{L W} \approx \sigma^{*}\left(T_{\mathrm{srf}} k^{*}-T_{\mathrm{cld}}^{k^{*}}\right)\left(1-e^{-\delta^{*} \tau}\right)$,

with $\sigma^{*}=1.607 \times 10^{-4} \mathrm{~W} \mathrm{~m}^{-2} \mathrm{~K}^{-4}, \quad k^{*}=2.528 \quad$ and $\delta^{*}=0.75$.

According to Eq. (5), $\mathrm{CRF}^{L W}$ is proportional to the difference between ground and cloud top temperature. $\mathrm{CRF}^{L W}$ is usually positive, because the ground is typically warmer than the top of the cloud. $\mathrm{CRF}^{L W}$ is also proportional to the cloud emissivity $\epsilon$. A comparison to the $\mathrm{CRF}^{L W}$ calculated with the radiative transfer model reveals a mean error of $10 \%$ in the estimated $\mathrm{CRF}^{L W}$ based on Eq. (5).

For optically thin clouds $(\tau<0.3)$, we can linearize Eq. (6) and arrive at

$\mathrm{CRF}^{L W} \approx \sigma^{*}\left(T_{\text {srf }}^{k^{*}}-T_{c l d}^{k^{*}}\right) \delta^{*} \tau$.

This equation will be useful in the discussion of the net cloud radiative forcing (Sect. 3.3).

\subsection{Shortwave radiative budget}

Complementary to the longwave radation, the shortwave radiation comprises the visible and near-visible portions of the electromagnetic spectrum with wavelengths ranging from

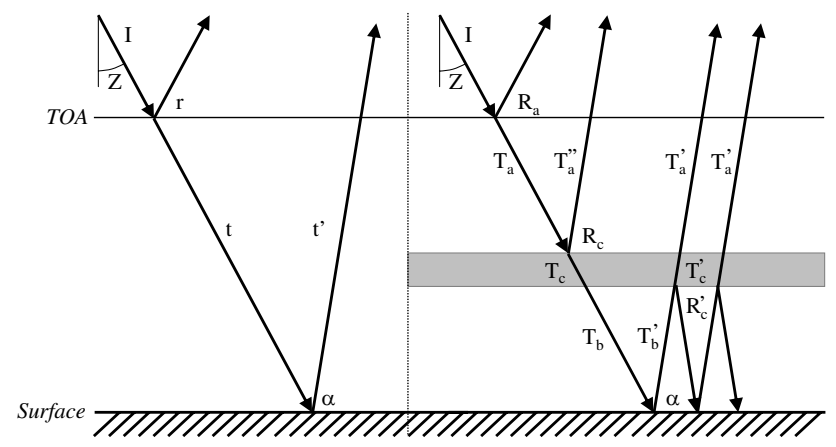

Fig. 3. Plane parallel model for the shortwave radiative budget for clear sky (left) and cloudy sky (right). $I$ is the incident solar flux with solar zenith angle $Z$. $r$ and $R$ stand for reflectance, $t$ and $T$ for transmittance. $\alpha$ is the surface albedo.

0.4 to $4 \mu \mathrm{m}$, which is essentially of solar origin (Glickman, 2000).

The shortwave radiative budget is more complex than the longwave budget, because it deals with incoming and outgoing fluxes. Figure 3 shows a schematic of the plane parallel model that we want to discuss here, closely following Chylek and Wong (1998). The clear sky situation is depicted on the left hand side of Fig. 3. For a given daytime and location, the incident solar flux $I$ depends on the solar zenith angle $Z, I=S \cos (Z)$, where $S$ is the solar constant. $r$ denotes the reflectivity of the atmosphere. The direct incident beam crosses the atmosphere with a transmittance $t$ and is reflected at the surface with an reflectivity (albedo) $\alpha$. The transmittance for the outgoing diffuse beam is $t^{\prime}$. The TOA clear sky shortwave net flux $F_{\mathrm{clr}}^{S W}$ is then

$F_{\mathrm{clr}}^{S W}=I\left(1-r-t t^{\prime} \alpha\right)$.

The cloudy sky situation is described on the right hand side of Fig. 3, forming a three layer plane parallel model of the atmosphere. The reflectance of the layer above the cloud is $R_{a}$. All layers have a specific transmittance for the downward direct beam and for the diffuse radiation. The reflectance of the cloud is $R_{c}$ for the incoming beam and $R_{c}{ }^{\prime}$ for the outgoing radiation.

If we take multiple reflections between the cloud and the surface into account and neglect all other multiple reflections between individual layer, then the TOA cloudy sky shortwave net flux $F_{\text {cld }}^{S W}$ becomes

$$
\begin{aligned}
& F_{\mathrm{cld}}^{S W}=I\left(1-R_{a}-R_{c} T_{a} T_{a}{ }^{\prime \prime}-\alpha T_{a} T_{a}{ }^{\prime} T_{b} T_{b}^{\prime} T_{c} T_{c}{ }^{\prime}\right.
\end{aligned}
$$

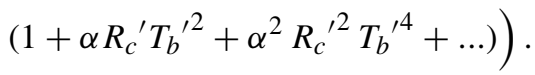

This equation has a considerable number of unknown parameters so that we have to make some simplifications before we continue. Let us assume that the reflectance and two-way transmittance of the atmospheric layer above the 
cloud are the same in cloudy as the in clear sky, $R_{a}=r$ and $T_{a} T_{a}{ }^{\prime}=T_{a} T_{a}{ }^{\prime \prime}=t t^{\prime}$. This means that we assume all atmospheric reflection and absorption to occur above the cloud. Furthermore, we assume that no absorption is occurring in the atmospheric layer below the cloud and thus $T_{b}=T_{b}{ }^{\prime}=1$. Finally, the cloud layer is assumed to be non absorbing in the shortwave spectrum, so that $T_{c}=1-R_{c}$. We then obtain after some rearrangement

$$
F_{\text {cld }}^{S W} \approx I\left(1-r-t t^{\prime} \alpha-(1-\alpha) t t^{\prime} \frac{R_{c}-\alpha R_{c}{ }^{\prime}}{1-\alpha R_{c}{ }^{\prime}}\right) .
$$

The reflectivity of the cloud layer can be estimated using (Baker, 1997),

$$
R_{c}=\frac{\tau}{\gamma+\tau}
$$

where $\gamma=1 /(1-g)$ and $g$ is the asymmetry factor, a measure for the fraction of light scattered forward by the cloud particles. However, Eq. (10) only refers to vertical incidence. To calculate $R_{c}$ and $R_{c}^{\prime}$, we have to consider that the shortwave radiation is not necessarily propagating vertically to the cloud. The effective cloud optical depth for the incident direct beam of solar radiation depends on the cosine of the zenith angle $\zeta=\cos (Z)$ and is approximately $\tau_{\text {eff }}=\tau / \zeta$, so that

$$
R_{c} \approx \frac{\tau / \zeta}{\gamma^{*}+\tau / \zeta}
$$

This equation is a rough approximation that is only accurate at small optical depth in its original form (Coakley and Chylek, 1975). We will therefore use radiative transfer calculations to estimate an optimum value for $\gamma^{*}$ and thus make the equation applicable to a larger range of optical depths. We could further expand our parameterization by deriving a function for $\gamma^{*}$. The use of a fixed value leads however to a acceptable accuracy of our parameterization (see below) and keeps it as simple as possible.

The upward beam of shortwave radiation consists of diffuse radiation. Under the assumption that the radiation is isotropic, the reflectivity $R_{c}{ }^{\prime}$ is to a good approximation

$R_{c}^{\prime} \approx \frac{2 \tau}{\gamma^{*}+2 \tau}$.

By means of radiative transfer calculations using the $\mathrm{Fu}-$ Liou code, we find $\gamma^{*}=7.7$.

We can deduce typical values for the two-way transmissivity $t t^{\prime}$ from the clear sky radiative transfer calculations used in the previous section. $t t^{\prime}$ depends on the solar zenith angle and the atmospheric composition. For the sake of simplicity, we will use a mean value here. For diurnal mean conditions, a good value is $t t^{\prime *}=0.73$.

We obtain the shortwave cloud radiative forcing $\left(\mathrm{CRF}^{S W}\right)$ as the difference of Eqs. (9) and (7):

$\mathrm{CRF}^{S W}=F_{\mathrm{cld}}^{S W}-F_{\mathrm{clr}}^{S W} \approx-I t t^{\prime *}(1-\alpha) \frac{R_{c}-\alpha R_{c}{ }^{\prime}}{1-\alpha R_{c}{ }^{\prime}}$.
In comparison to the radiative transfer calculations, this parameterization of $\mathrm{CRF}^{S W}$ has a mean error of $7 \%$.

In the remainder of this section we discuss some specific cases of Eq. (13).

In case of $\alpha=1, \mathrm{CRF}^{S W}$ becomes zero, as the perfect ground reflectivity is not increased by the addition of a cloud in this case. In the opposite case, $\alpha=0$, there are no multiple reflections between cloud and ground. Equation (13) then becomes $\mathrm{CRF}^{S W} \approx-I t t^{\prime *} R_{c}$, thus maximizing $\mathrm{CRF}^{S W}$.

Equation (13) differs from similar expressions (Paltridge and Platt, 1976; Twomey, 1977). These assume that $R_{c}$ and $R_{c}{ }^{\prime}$ are equal, which is not generally the case. Furthermore, our expression describes an interesting phenomenon. We can conclude from Eqs. (11) and 12) that for solar zenith angles below $60^{\circ}$, the cloud reflectivity is smaller for the incoming than for the outgoing radiation, i.e., $R_{c}<R_{c}{ }^{\prime}$. As a consequence, part of the solar radiation can become trapped between cloud and surface, leading to a positive $\mathrm{CRF}^{S W}$. It becomes clear from Eq. (13) that this is only the case for high surface albedo. This finding is in accordance with a more detailed study by Carlin et al. (2002).

For optically thin clouds, we can linearize the equations for $R_{c}$ and $R_{c}{ }^{\prime}$, yielding

$\mathrm{CRF}^{S W} \approx-I \frac{t t^{\prime *}}{\gamma^{*}}(1-\alpha)\left(\frac{1}{\zeta}-2 \alpha\right) \tau$.

This equation is applicable for effective optical depths $(\tau / \zeta$ and $2 \tau)$ below 0.6 .

Often, we are interested in the CRF for daily mean conditions. As a convenient approximation (see e.g., Hartmann et al., 2001), we can calculate the daily mean solar zenith angle $\bar{Z}$ by averaging over its cosine during daytime. The daily mean incident solar flux $\bar{I}$ is then

$\bar{I}=S f \cos (\bar{Z})$,

where $f$ is the fraction of the day that the sun is above the horizon.

\subsection{Net cloud radiative forcing}

We can now easily compute the net cloud radiative forcing $\left(\mathrm{CRF}^{\text {net }}\right)$ by adding $\mathrm{CRF}^{L W}$ to $\mathrm{CRF}^{S W}$, using Eqs. (5) and (13). In comparison with the results from the radiative transfer model, the accuracy of our parameterization is typically better than $20 \%$. The parametrization also compares favourably with Meerkotter et al. (1999), as shown in Schumann (2009).

The combination of Eqs. (5) and (13) does not lead any further in terms of a mathematical analysis. For optical depths below about 0.3, we can alternatively use Eqs. (6) and (14), yielding

$\mathrm{CRF}^{\text {net }} \approx$

$\left(\left(\sigma^{*} \delta^{*} T_{\mathrm{srf}}^{k^{*}}-T_{\mathrm{cld}}^{k^{*}}\right)-I \frac{t t^{\prime *}}{\gamma^{*}}(1-\alpha)\left(\frac{1}{\zeta}-2 \alpha\right)\right) \tau$. 
In this limit, $\mathrm{CRF}^{\text {net }}$ depends linearly on $\tau$. Furthermore, we can easily derive the condition for $\mathrm{CRF}^{\text {net }}$ equaling zero, which satisfies the equation

$T_{\mathrm{srf}}{ }^{k^{*}}-T_{\text {cld }} k^{*}=787 I(1-\alpha)\left(\frac{1}{\zeta}-2 \alpha\right)$,

where we have inserted the values $t t^{\prime *}, \gamma^{*}, \sigma^{*}$ and $\delta^{*}$. For the mean tropical conditions discussed in the next section $\left(T_{\text {srf }}=299 \mathrm{~K}, I=435 \mathrm{Wm}^{-2}, \zeta=0.636, \alpha=0.05\right)$, Eq. (17) yields a critical cloud top temperature $T_{\text {cld }}$ of $265 \mathrm{~K}$, corresponding to $6.25 \mathrm{~km}$ altitude in the tropics.

\section{Sample application - tropical cirrus}

We are now in a position to estimate CRF for any atmospheric condition with a cloudiness that can be approximated by a single cloud layer. Here, we apply the parameterization to the example of tropical cirrus clouds and compare the results to calculations with the radiative transfer model for typical tropical conditions (see Sect. 2). In accordance to the profile used for the radiative transfer calculations, we set the surface temperature $T_{\text {srf }}$ to $299 \mathrm{~K}$, the surface albedo to 0.05 , and assume the solar radiation as found in daily mean equinox conditions at the equator $\left(I=435 \mathrm{Wm}^{-2}\right.$ and $\left.\zeta=0.636\right)$.

Figure 4 shows estimated CRF (contour lines) for cirrus clouds under tropical conditions based on Eqs. (5) and (13). Cloud top temperatures are translated to cloud top altitudes according on the mean tropical temperature profile. For comparison, the color shadings indicate differences to CRF calculated with the radiative transfer model.

Panel a shows estimated $\mathrm{CRF}^{L W}$ using Eq. (5). The differences for clouds above $10 \mathrm{~km}$ altitude remain below a combined absolute and relative error of $5 \mathrm{~W} \mathrm{~m}^{-2}$ and $6 \%$, respectively. In the light of the simplicity of our parametrization, the agreement between the estimated and calculated forcing is quite good.

Analogously, $\mathrm{CRF}^{S W}$ is shown in Fig. 4b. Because we have no dependency on the vertical position of the cloud in our parametrization, the estimated $\mathrm{CRF}^{S W}$ depends on the cloud optical depth only. The color shadings represent differences to $\mathrm{CRF}^{S W}$ calculated with the radiative transfer model using the same atmospheric profiles. The absolute differences remain below $5 \mathrm{~W} \mathrm{~m}^{-2}$ for clouds above $10 \mathrm{~km}$ altitude. The overall agreement between estimated and calculated values is very good.

Adding $\mathrm{CRF}^{L W}$ to $\mathrm{CRF}^{S W}$, we arrive at the net cloud radiative forcing $\left(\mathrm{CRF}^{\text {net }}\right)$, which is shown in Fig. $4 \mathrm{c}$. For small optical depths, $C R F^{\text {net }}$ is negative below about $6 \mathrm{~km}$ and positive above. This altitude is in agreement with Eq. (17) (see previous section). For denser clouds, the transition from negative to positive $\mathrm{CRF}^{\text {net }}$ occurs at a higher altitude. The overall agreement between estimated and calculated $\mathrm{CRF}^{\text {net }}$ is again quite good.
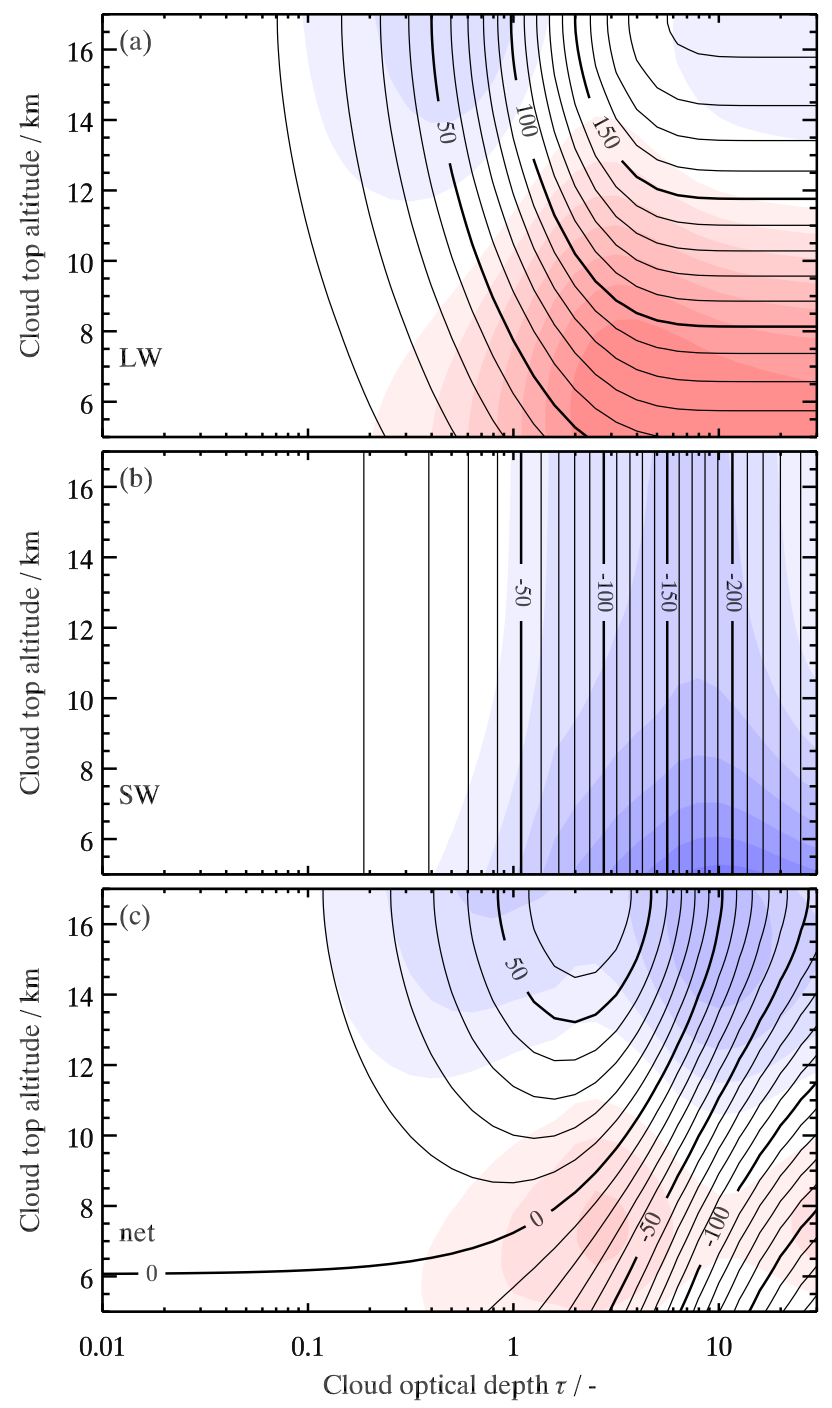

Fig. 4. Contours: Estimated daily mean cloud radiative forcing (CRF) in $\mathrm{Wm}^{-2}$ as functions of cloud top altitude and cloud optical depth for tropical conditions. Shadings: Differences to calculated CRF for the same conditions using the radiative transfer model in steps of $2 \mathrm{Wm}^{-2}$. Positive differences are indicated by red shadings.

The largest absolute differences to the radiative transfer calculations are at high altitude and optical depths of about 10 , right where the transition from cooling to heating occurs. In this area, $\mathrm{CRF}^{\text {net }}$ is also sensitive to changes in optical depth. The difference to the radiative transfer calculations is equivalent to an overestimation of the could optical depth by $10 \%$.

Cirrus clouds typically have cloud top temperatures below $238 \mathrm{~K}$ (Sassen, 2002), which corresponds to altitudes above $10 \mathrm{~km}$ in the mean tropical atmosphere. Moreover, Sassen (2002) defines cirrus clouds as ice clouds with optical depth 


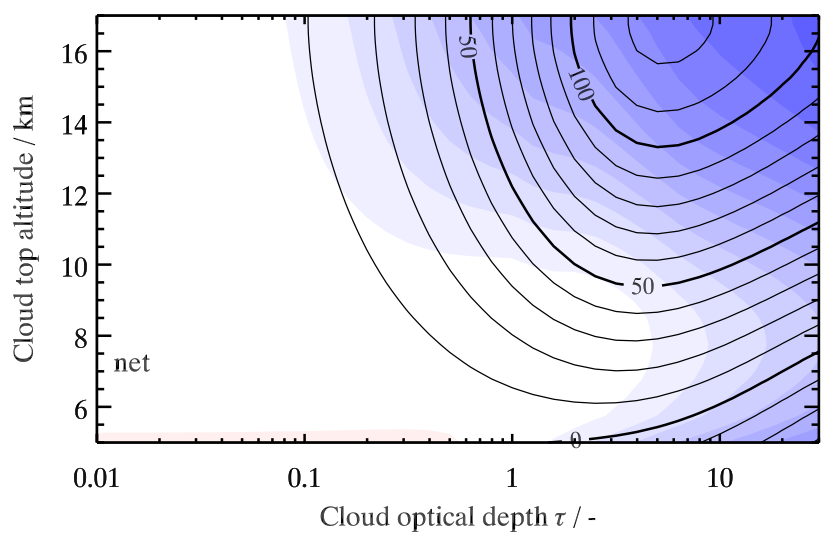

Fig. 5. Contours: Estimated net cloud radiative forcing $\left(\mathrm{CRF}^{\text {net }}\right)$ in $\mathrm{Wm}^{-2}$ for the case of an underlying cloud with optical depth 20 and cloud top temperature $0^{\circ} \mathrm{C}$. Shadings: Differences to radiative transfer calculations in steps of $2 \mathrm{Wm}^{-2}$. The blue shadings indicate negative differences.

below 3.0. We can thus see from Fig. 4 that tropical cirrus clouds always have a positive $\mathrm{CRF}^{\text {net }}$, i.e., that the infrared effect is dominating.

So far, we have considered the radiative effect of a cloud in otherwise cloud free conditions. But, what is the radiative effect of a cirrus cloud in the presence of underlying clouds? Because lower clouds usually have considerably higher optical depths than cirrus clouds, it is quite obvious to use our parametrization to examine this case by setting the surface temperature and albedo to the cloud top temperature and reflectivity of the underlying cloud. Here, we discuss the case of a tropical cirrus cloud above a cumulus congestus cloud with a typical cloud top temperature of $0{ }^{\circ} \mathrm{C}$ (Johnson et al., 1999). Figure 5 shows the estimated radiative effect of a cloud in the presence of an underlying cumulus congestus relative to the situation including only the underlying cloud. The optical depth of the underlying cloud was assumed to be 30, resulting in a cloud reflectivity of 0.8 (from Eq. 11). A comparison with Fig. 4 shows that the cloud radiative effect of the high cloud is essentially a reduced infrared effect, whereas the shortwave effect comes only into play for the highest optical depths, where the optical depth of the high cloud increases the total reflectivity.

The shadings represent the differences to radiative transfer calculations including a $2 \mathrm{~km}$ thick underlying cloud with a cloud top temperature of $0^{\circ} \mathrm{C}$ and an optical depth of 30 . The agreement is very good if we consider that we have twisted our simple parametrization to produce that result.

\section{Conclusions}

We have presented a simple model for the longwave and shortwave cloud radiative forcing (CRF).
The parameterization was derived from a set of radiative transfer calculations covering a global range of realistic atmospheric conditions and cloud properties, and thus has a wide range of applicability.

The accuracy of our semi-quantitative parameterization is typically better than $20 \%$ when comparing with the accurate results from the $\mathrm{Fu}$ and Liou $(1992,1993)$ radiative transfer model. Possible applications include the convenient estimate of CRF for a global range of environmental conditions, the evaluation of the sensitivity to changes in environmental conditions, and as a tool in education. To support the use of our parameterization, we offer an online version of the model at http://www.iac.ethz.ch/url/crf.

Acknowledgements. This work was partly supported by the EU projects TROCCINOX and SCOUT-O3 through the Swiss Federal Office for Education and Science. ERA 40 data have been provided by ECMWF.

Edited by: N. Harris

\section{References}

Allan, R. P., Shine, K. P., Slingo, A., and Pamment, J. A.: The dependence of clear-sky outgoing long-wave radiation on surface temperature and relative humidity, Q. J. Roy. Meteor. Soc., 125, 2103-2126, 1999.

Baker, M. B.: Cloud microphysics and climate, Science, 276, 10721078, 1997.

Barkstrom, B. R.: The Earth Radiation Budget Experiment (Erbe), B. Am. Meteor. Soc., 65, 1170-1185, 1984.

Carlin, B., Fu, Q., Lohmann, U., Mace, G. G., Sassen, K., and Comstock, J. M.: High-cloud horizontal inhomogeneity and solar albedo bias, J. Clim., 15, 2321-2339, 2002.

Chen, T., Rossow, W. B., and Zhang, Y. C.: Radiative effects of cloud-type variations, J. Clim., 13, 264-286, 2000.

Chylek, P. and Wong, J. G. D.: Cloud radiative forcing ratio - An analytical model, Tellus A, 50, 259-264, 1998.

Coakley, J. A. and Chylek, P.: The 2-stream approximation in radiative transfer - including angle of incident radiation, J. Atmos. Sci., 32, 409-418, 1975.

Corti, T., Luo, B. P., Peter, T., Vomel, H., and Fu, Q.: Mean radiative energy balance and vertical mass fluxes in the equatorial upper troposphere and lower stratosphere, Geophys. Res. Lett., 32, L06802, doi:10.1029/2004GL021889, 2005.

$\mathrm{Fu}, \mathrm{Q}$. and Liou, K. N.: On the Correlated K-Distribution Method for Radiative-Transfer in Nonhomogeneous Atmospheres, J. Atmos. Sci., 49, 2139-2156, 1992.

$\mathrm{Fu}, \mathrm{Q}$. and Liou, K. N.: Parameterization of the Radiative Properties of Cirrus Clouds, J. Atmos. Sci., 50, 2008-2025, 1993.

Fu, Q., Yang, P., and Sun, W. B.: An accurate parameterization of the infrared radiative properties of cirrus clouds for climate models, J. Clim., 11, 2223-2237, 1998.

Fu, Q. A.: An accurate parameterization of the solar radiative properties of cirrus clouds for climate models, J. Clim., 9, 2058-2082, 1996.

Glickman, T. S.: Glossary Of Meteorology, American Meteorological Society, 2nd ed., 855 pp., 2000. 
Hartmann, D. L., Ockertbell, M. E., and Michelsen, M. L.: The Effect of Cloud Type on Earths Energy-Balance - Global Analysis, J. Clim., 5, 1281-1304, 1992.

Hartmann, D. L., Holton, J. R., and Fu, Q.: The heat balance of the tropical tropopause, cirrus, and stratospheric dehydration, Geophys. Res. Lett., 28, 1969-1972, 2001.

Heymsfield, A. J. and Platt, C. M. R.: A Parameterization of the Particle-Size Spectrum of Ice Clouds in Terms of the AmbientTemperature and the Ice Water-Content, J. Atmos. Sci., 41, 846855, 1984.

IPCC: Climate Change 2007 - The Physical Science Basis, Cambridge University Press, 996 pp., 2007.

Johnson, R. H., Rickenbach, T. M., Rutledge, S. A., Ciesielski, P. E., and Schubert, W. H.: Trimodal characteristics of tropical convection, J. Clim., 12, 2397-2418, 1999.

Liou, K.: Radiation and Cloud Processes in the Atmosphere, Oxford University Press, 1992.

Meerkotter, R., Schumann, U., Doelling, D. R., Minnis, P., Nakajima, T., and Tsushima, Y.: Radiative forcing by contrails, Ann. Geophys.-Atmos. Hydrospheres Space Sci., 17, 10801094, 1999.
Paltridge, G. W. and Platt, C. M. R., Radiative Processes in Meteorology and Climatology, Elsevier, Amsterdam, Netherlands, 1976.

Raval, A., Oort, A. H., and Ramaswamy, V.: Observed Dependence of Outgoing Longwave Radiation on Sea-Surface Temperature and Moisture, J. Clim., 7, 807-821, 1994.

Rossow, W. B. and Lacis, A. A.: Global, Seasonal Cloud Variations from Satellite Radiance Measurements, 2. Cloud Properties and Radiative Effects, J. Clim., 3, 1204-1253, 1990.

Sassen, K.: Cirrus Clouds, in: Cirrus, edited by: Lynch, D. K., Sassen, K., Starr, D. O., and Stephens G., Oxford University Press, 231-255, 2002.

Schumann, U.: Interactive comment on "A simple model for cloud radiative forcing" by T. Corti and T. Peter, Atmos. Chem. Phys. Discuss., 9, C2142-C2143, 2009.

Stephens, G. L., Tsay, S. C., Stackhouse, P. W., and Flatau, P. J.: The Relevance of the Microphysical and Radiative Properties of Cirrus Clouds to Climate and Climatic Feedback, J. Atmos. Sci., 47, 1742-1753, 1990.

Twomey, S.: Atmospheric Aerosols, Elsevier, Amsterdam, The Netherlands, 302 pp., 1977. 